\title{
Educación para la paz como estrategia pedagógica para la prevención de la violencia escolar
}

\author{
Peace education as a pedagogical strategy for the prevention of school \\ violence
}

\author{
Raúl Filomeno Zuna Llanos \\ razulla@hotmail.com \\ Universidad Autónoma “Tomás Frías”, Potosí, Bolivia
}

\section{RESUMEN}

La violencia escolar es un tema que preocupa a la sociedad, por lo tanto la prevención de este fenómeno es de interés para todos los involucrados. El propósito de este estudio es el abordaje de la prevención de la violencia escolar en la Educación Secundaria Comunitaria Productiva por lo cual se concibe la educación para la paz como la estrategia pedagógica que permita lograr una cultura educativa basada en los valores y Derechos Humanos. Se fundamenta en un diagnóstico de necesidades para lo cual se utilizaron diversas técnicas e instrumentos que permitieron recoger la percepción de 150 estudiantes, 15 docentes, 70 padres y los directivos de una Unidad Educativa en Potosí. Los resultados indican que existe la necesidad de formación en prevención de violencia escolar de todos los participantes. La propuesta se diseña fundamentada en talleres prácticos que permiten el análisis crítico y la reflexión de los aspectos que generan la violencia en la escuela y la práctica de acciones para capacitar en el abordaje de la prevención de la violencia en el contexto educativo.

Palabras clave: Educación para la paz, violencia escolar, estrategia pedagógica, educación secundaria

\section{ABSTRACT}

School violence is an issue that concerns society, therefore the prevention of this phenomenon is of interest to all involved. The purpose of this study is the approach to the prevention of school violence in Productive Community Secondary Education, which is why education for peace is conceived as the pedagogical strategy that allows to achieve an educational culture based on Human Rights and values. It is based on a diagnosis of needs for which various techniques and instruments were used that allowed to collect the perception of 150 students, 15 teachers, 70 parents and the directors of an Educational Unit in Potosí. The results indicate that there is a need for training in prevention of school violence of all participants. The proposal is designed based on practical workshops that allow critical analysis and reflection of the aspects that generate violence in school and the practice of actions to train in addressing violence prevention in the educational context.

Key words: Peace education, school violence, pedagogical strategy, secondary education 


\section{INTRODUCCIÓN}

La violencia escolar es una problemática que por sus manifestaciones y consecuencias genera preocupación en las distintas esferas de la sociedad como: el Estado, las instituciones educativas, la comunidad y la familia. García y Madriaza (2005), Ortega (2001) y Oteros (2006) caracterizan la violencia escolar como las situaciones que se generan en el contexto educativo por relaciones interpersonales en las cuales se causa daño no sólo a la víctima sino también sufre daño el agresor.

Por su complejidad el tema de la prevención de la violencia escolar es un desafío en la búsqueda del desarrollo de alternativas factibles y efectivas que puedan dar respuesta para resolver esta situación. El Estado genera leyes para el apoyo de las medidas contra la violencia escolar como lo evidencia el Decreto Presidencial del Estado Plurinacional de Bolivia №1302 (2012) en el cual se responsabiliza directamente al Ministerio de Educación en el desarrollo de un plan específico para enfrentar la violencia, maltrato y abuso en el ámbito educativo.

La educación como fenómeno social es determinante, porque a través de ella el ser humano desarrolla sus capacidades, habilidades, conocimientos y estructura las normas para la convivencia. En consecuencia la Escuela tiene que vincularse estrechamente con los demás agentes educativos como: la familia, la comunidad, las instituciones y organizaciones, para atender y solucionar los conflictos de forma pacífica y asegurar la armonía social a nivel grupal e interpersonal en el ámbito educativo. Para este propósito se debe educar y formar a las personas a cualquier edad, para que sean capaces, de comprender, enfrentar y resolver los problemas, de forma razonada, reflexionada, crítica y consciente.
El papel de la educación es muy importante porque se convierte en el medio para atender y dar respuesta, ya que es en el contexto educativo donde se presenta la violencia como problemática. Por consiguiente para entender la violencia escolar se debe estudiar desde la realidad en la cual se está originando y con los actores involucrados: personal docente, personal directivo, estudiantes y familia. La comprensión del fenómeno de violencia escolar guiará las acciones más efectivas para minimizarla en el contexto educativo. Las propuestas fundamentadas en la convivencia el respeto y la paz son las que se estiman como las más positivas para atender la situación de violencia en el contexto escolar.

Como sustento teórico para enmarcar el estudio se seleccionó el modelo ecológico ya que promueve la participación dinámica y comprometida de todos los actores involucrados en el quehacer educativo. Este enfoque es el que muestra mayor evidencia empírica con resultados positivos para minimizar el clima de violencia; se basa en la necesidad de promover una preparación de carácter comunitario que implica procesos de aprendizajes y enseñanzas de forma continua para generar un nuevo estilo de vida basado en una cultura de paz.

El enfoque ecológico para la prevención de la violencia toma en cuenta los complejos niveles de la interacción entre el individuoambiente, es decir, que la conducta relacionada con cualquier fenómeno de índole natural o social es resultado de la interacciones entre los individuos y su entorno. Por tanto las conductas personal y colectiva dependen de la sociedad y de sus subsistemas, depende de una compleja red de interrelaciones que se establecen en su interior, porque es allí donde deben buscarse las intervenciones. (Bronfenbrenner, 1987). Se establecen de esta manera relaciones 
interpersonales entre los estudiantes, los docentes, los padres de familia y el personal directivo.

Este enfoque busca disminuir las condiciones de riesgo, incrementando los factores protectores en los complejos niveles de la interacción individuo - ambiente, mediante el fomento de competencias $y$ fortalezas de los estudiantes y el fomento de un clima satisfactorio para los participantes del contexto escolar, esto incluye los escenarios en los que transcurre su vida (escolar, familiar, de ocio), las conexiones entre dichos escenarios, los medios de comunicación y el conjunto de creencias sociales.

A partir de todos estos elementos el enfoque ecológico, plantea que la prevención de la violencia escolar solo podrá realizarse fortaleciendo las relaciones entre los microsistemas que constituyen la comunidad educativa, dentro y fuera de la clase, o sea, en las conversaciones, los juegos, la cooperación, la competición y el trabajo, para mantener un clima escolar adecuado y que permita apropiarse de su futuro. (Díaz Aguado, 2002). Para ello será necesario: promover climas adecuados generar bienestar, educar en valores y cambio de actitudes hacia la violencia, potenciar la participación, fortalecer las relaciones sociales de cooperación, promover estrategias conductuales para hacer contrarrestar a la violencia a partir de sus potencialidades (Rodney, 2010).

La prevención de la violencia escolar debe promover cambios significativos desde la organización administrativa, y los actores educativos, donde se establezca espacios y entornos cooperativos, lo que implica la preparación de los sujetos de la educación; para lograr tal fin se deben establecer proyectos educativos que fomenten la prevención de la violencia escolar a partir de estrategias metodológicas y programas de prevención basados en la participación responsable de todos involucrados (Díaz Aguado, 2005).

La prevención de la violencia desde el enfoque ecológico se complementa con el paradigma socio comunitario. Este paradigma se orienta hacia un trabajo, grupal, integrado y holístico; donde los actores educativos puedan inmiscuirse en el propósito de la educación como sujetos activos, para lograr un verdadero proceso que articule la práctica pedagógica con la práctica comunitaria.

La educación por tanto tiene sentido en la medida que sea capaz de incidir, transformar y comprometerse con los problemas y necesidades en la que está inmiscuida la escuela; desde esta visión la educación ya no se reduce a la mera enseñanza - aprendizaje o al trabajo de aula, sino a desarrollar la educación desde la vida y para la vida, razón por la cual promueve la intra y la interculturalidad, a partir de reconocimiento y promoción de los conocimientos locales y los conocimientos universales que dan lugar a la construcción de nuevos conocimientos, a partir de un proceso de dialógico, de reflexión y acción transformadora (Ministerio de Educación, 2015) lo que permite construir un nuevo paradigma del Vivir Bien (Huanacuni, 2010), principio constitucional que no se reduce a lo individual, sino que trasciende e involucra a la comunidad, la misma que es entendido como la convivencia armónica entre la vida de uno, la vida del otro, y la vida de la naturaleza y la madre tierra( Ministerio de Educación, 2015, Mora-Merchán, 2001).

En consecuencia el trabajo preventivo de la violencia escolar es responsabilidad de toda la comunidad, entendiendo la misma como una unidad de convivencia de varios sujetos educativos porque coexisten, estas son: El personal docente, que constituye una 
unidad en sí un microsistema, aunque ésta no sea homogénea, pero que adquiere entidad diferenciada respecto de los otros actores educativos. Los estudiantes, además de ser el eje sobre el que gira toda la actividad educativa, se constituye como un sistema social diferenciado, cuya participación en la organización del centro, en la elaboración de las normas y en la asunción de principios y valores, es fundamental. Las familias que la Ley las constituyen en los actores internos de la escuela, con los cuales se establecen relaciones profundas y cercanas en los procesos educativos, porque permiten cooperar en el logro de los objetivos educativos.

Se considera que la prevención de las actitudes violentas debe ser el marco de referencia en el campo educativo. Por tal razón se asume la prevención de la violencia escolar desde los parámetros de la Educación para la paz. Educar para la paz implica utilizar de forma adecuada el poder $y$ poseer competencias para aplicar estrategias que permitan establecer normas, resolver conflictos de forma pacífica y civilizada. Para contribuir a este propósito, es indispensable que los estudiantes y los actores educativos de incidencia como los profesores y padres de familia, se formen $y$ se preparen en intervención y ante todo en prevención de la violencia escolar y puedan contribuir en el desarrollo de la personalidad de las nuevas generaciones para incidir en la construcción de entornos de convivencia armónica y de paz. (UNESCO, 2016). EL Estado Plurinacional de Bolivia, desde el año 2009, en la Nueva Constitución, asume esta postura del movimiento pacifista, declarando en su texto constitucional como un "Estado pacifista, que promueve la cultura de la paz y el derecho a la paz". (Constitución Política del Estado, 2009 art. 10 R. I), lo que muestra que existe una toma de conciencia para forjar una cultura de paz basada en la convivencia, el respeto y la tolerancia.

Al respecto el Ministerio de Educación y Ciencias de España concibe la Educación para la Paz como un proceso educativo, continuo y permanente, que se da a través de la aplicación de métodos problematizadores, que pretende desarrollar un nuevo tipo de cultura, la cultura de la paz, que ayude a las personas a develar críticamente la realidad, compleja y conflictiva, para poder situarse ante ella y actuar en consecuencia.

Una de las estrategias pedagógicas que permite potenciar las capacidades y recursos propios de una persona es el taller educativo, porque promueve nuevos aprendizajes desde la vivencia de nuevos contenidos y ofrece la posibilidad de ponerlos en práctica. (Díaz Barriga, 2002). Los talleres educativos pueden estar destinados a poblaciones de diferentes edades cronológicas, y con diferente propósito, su implementación es siempre de carácter grupal, sin embargo puede existir la posibilidad de trabajos individuales de reflexión y autocrítica. El taller como estrategia educativa permite la participación consciente y activa de los participantes promoviendo el aporte colectivo, la reflexión, el debate y la evaluación (Torrego y Negro, 2012).

Esta investigación considera que lograr integrar tanto la escuela a través de la formación de los profesores así como de la comunidad a través de los padres de familia, implica entender que la educación no es un hecho aislado de la comunidad sino que está articulada a ella tanto en el modo de aprendizaje como en el sentido, la finalidad y los participantes de la educación (Ministerio de educación, Unidad de Formación 1 Modelo Educativo Socio Comunitario Productivo, 2015). La implementación de un sistema de talleres tiene pertinencia pedagógica de formación de los maestros y padres de familia 
en prevención de la violencia desde la educación para la paz. Porque esta formación deberá ser trabajada de manera práctica con los estudiantes en una institución de Educación Secundaria Socio Comunitaria Productiva en la ciudad de Potosí en Bolivia. Después de lo anteriormente planteado se enuncian los objetivos que encontró este estudio.

\section{Objetivo General}

Diseñar una estrategia pedagógica para la prevención de la violencia escolar desde la educación para la paz dirigida a estudiantes del tercer año de Educación Secundaria Comunitaria Productiva

\section{Objetivos Específicos}

- Describir la formación del personal docente del tercer año de Educación Secundaria Comunitaria Productiva en estrategias para la prevención de la violencia escolar desde la educación para la paz

- Caracterizar la violencia escolar en los estudiantes del tercer año de Educación Secundaria Comunitaria Productiva

- Identificar la formación de los estudiantes, del tercer año de Educación Secundaria Comunitaria Productiva, en estrategias para la prevención de la violencia escolar desde la educación para la paz

- Determinar la formación de los padres, de los estudiantes del tercer año de Educación Secundaria Comunitaria Productiva, en estrategias para la prevención de la violencia escolar desde la educación para la paz

- Caracterizar los elementos teóricos y metodológicos para el diseño de la estrategia pedagógica para la prevención de la violencia escolar desde la educación para la paz
- Establecer la factibilidad de aplicación de la estrategia pedagógica para la prevención de la violencia escolar desde la educación para la paz

\section{MÉTODO}

Se desarrolló una investigación de tipo proyectiva ya que su objetivo fue diseñar una propuesta como solución a un problema a partir de un diagnóstico de necesidades. (Hurtado, 2012). Para lo cual se identifican las necesidades y se definen las variables, se comprenden las causas y consecuencias, se identifican posibles soluciones y se elabora una propuesta capaz de producir los cambios. Su diseño orientada en el campo ya que los datos se obtuvieron directamente de los sujetos investigados en el ambiente natural donde ocurren los hechos en el contexto escolar (Niño Rojas, 2011).

La población estuvo conformada por los estudiantes del Tercero de Educación Secundaria Comunitaria, el personal directivo y los padres de familia de los estudiantes. El número de estudiantes fue de140 estudiantes distribuidos en cuatro paralelos. Los maestros fueron 15 quienes se desempeñan en el tercer año de escolaridad en las distintas áreas, estos docentes tienen entre15 hasta 32 años de servicio. Los padres de familia fueron 70 , esta muestra se autoseleccionó en relación a su disponibilidad para participar en la investigación.

Las técnicas se usaron fue la observación no participativa la cual permitió que el investigador percibiera el tipo de relaciones interpersonales en el contexto escolar. La Otra técnica fue la encuesta ya que se obtienen los datos realizando preguntas por escrito a los estudiantes, docentes y padres de familia acerca de los aspectos de la violencia escolar y las estrategias fundamentadas en la educación para la paz. 
Además la entrevista fue la técnica que permitió en obtener datos realizando preguntas de forma verbal al Director de la institución educativa.

Para la observación participativa se utilizó una guía de observación que tenía como objetivo develar la violencia escolar en el contexto educativo natural en el cual se desenvuelven los diferentes individuos. Esta guía está conformada por 19 ítems, para observar las relaciones interpersonales entre estudiantes, estudiantes - docentes, estudiantes directivos, directivos - docentes y la actitud que asumen acerca de la violencia escolar.

Para obtener información en forma escrita el instrumento que se utilizó fue el cuestionario el cual se presentó en varias versiones. La versión para el estudiante tiene 35 ítems, las versiones para los docentes y los padres 30 ítems. Estos cuestionarios tienen como función conocer la actitud hacia la violencia, detectar los tipos y frecuencia de los actos de violencia que ocurren en el contexto, los protagonistas de los hechos violentos y los mecanismos para afrontar la violencia. Adicionalmente para la factibilidad se pregunta acerca del interés y disposición a participar en la formación de estrategias para la prevención de la violencia escolar desde la educación para la paz.

El instrumento que permitió realizar la entrevista fue una guía de entrevista semiestructurada con 10 preguntas abiertas acerca de la temática en estudio.

Además el estudio comprendió la aplicación de varias fases como: el diagnóstico, la planificación y diseño de la propuesta.
- El diagnóstico permite indagar sobre los tipos de violencia que se desarrollan en el contexto de estudio, desde la realidad de sus protagonistas los estudiantes, docentes, directivo y padres de familia. Es un diagnóstico integral que determinará las necesidades educativas individuales y colectivas en relación con la prevención de la violencia escolar.

- La segunda fase es la de planificación, en la cual se organizan y analizan los resultados del diagnóstico y en función de éstos se toman decisiones acerca de la mejor forma de abordaje.

- La tercera fase es la del diseño, en esta se elabora la propuesta de la estrategia pedagógica para la prevención de la violencia escolar a través de la formación de los docentes, estudiantes y padres de familia.

\section{RESULTADOS}

\section{Presencia de violencia escolar}

En relación a la existencia de la violencia escolar, las autoridades en un 100\% consideran que existen manifestaciones de violencia en la escuela, sin embargo consideran que no salen a la luz pública todas sino las que son de gravedad o cuando alguna persona reclama; esta percepción es corroborada por los maestros quienes en un 93\% consideran que existe violencia, mientras los padres de familia solo en un $31.46 \%$ de los padres de familia considera la existencia de violencia en escolar, frente a un $68.5 \%$ que considera que no conoce o no tiene información sobre la existencia de violencia; mientras que un $79 \%$ de los estudiantes declaran que existe violencia escolar. (Ver tabla 1 y gráfico 1). 
Tabla 1. Reconocimiento de violencia escolar

\begin{tabular}{lcccc}
\hline & & \multicolumn{3}{c}{ \% Reconocimiento de la violencia escolar } \\
\hline & Directivos & Docentes & Padres & Estudiantes \\
& & & & \\
\hline Si existe & 100 & 93 & 31 & 79 \\
\hline No existe & & 7 & 69 & 21 \\
\hline
\end{tabular}

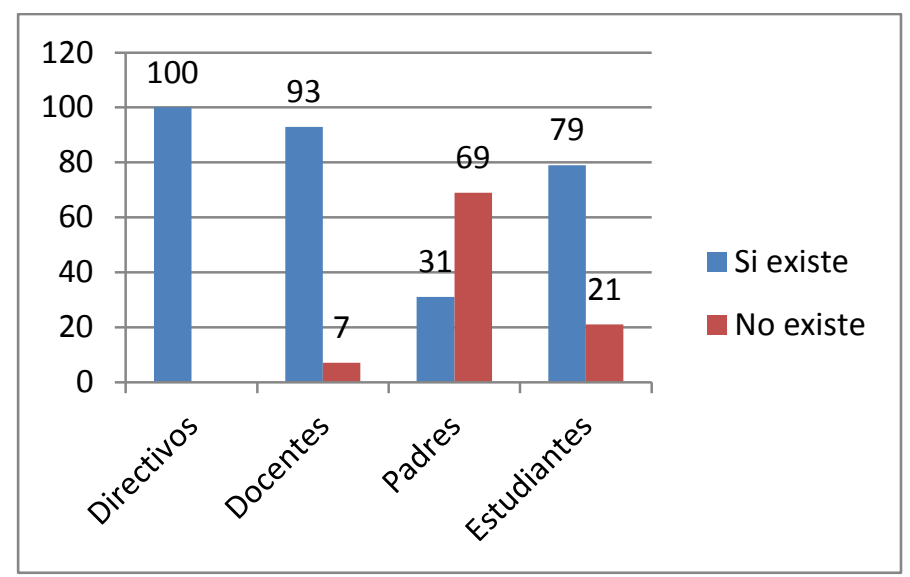

Gráfico 1. Reconocimiento de la violencia escolar

Los actores que se encuentran en convivencia en el contexto escolar están de acuerdo en declarar que existe la violencia, los padres de familia desconocen la violencia en mayor porcentaje.

La violencia se desarrolla en diferentes lugares o espacios de la escuela, es así que un $80 \%$ de los estudiantes y el $82 \%$ de los maestros consideran que el lugar de preferencia para que se originen actos de violencia es el patio y el $10 \%$ y $8 \%$ respectivamente declaran que es en el baño donde se desarrolla la violencia. El 100\% de las autoridades consideran el patio como el lugar en el cual se observa la violencia. El porcentaje restante está considerado en las aulas (Ver tabla 2 y gráfico 2 ).

Tabla 2. Lugares en los cuales es más frecuente la violencia escolar

\begin{tabular}{lccc}
\hline \multicolumn{4}{c}{ \% frecuencia de violencia escolar } \\
\hline & Directivos & Docentes & Estudiantes \\
Patio & 100 & 80 & 82 \\
\hline Baño & & 10 & 8 \\
\hline Aulas & & 6 & 5 \\
\hline Pasillos & & 4 & 5 \\
\hline
\end{tabular}




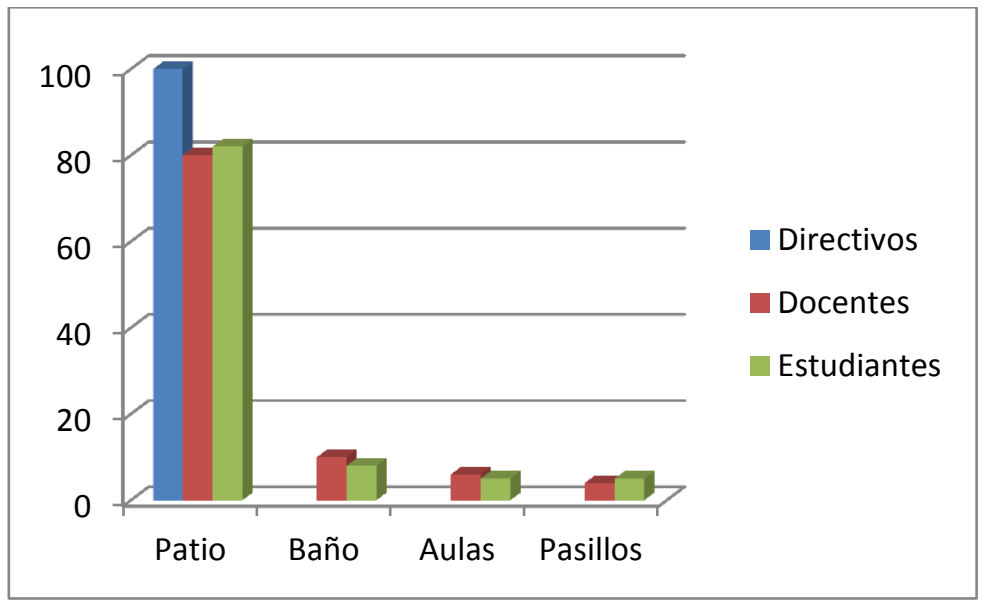

Gráfico 2. \% frecuencia de violencia escolar

Con respecto a la forma de la violencia la más frecuente es la violencia psicológica, a pesar de ser la más recurrente no se denuncia por lo tanto no quedan evidencias y en segundo lugar estaría la violencia física. La violencia de género no se visibiliza porque no hay denuncias.

A partir de la información obtenida se puede concluir que el problema de la agresión y victimización es propio de la escuela y de las relaciones interpersonales inter e intra género que se establecen entre el profesorado $\mathrm{y}$ el estudiantado fundamentalmente, se puede observar que todos los miembros de la comunidad educativa en algún momento se convierten en agresores o victimarios es también importante remarcar que la categoría relevantes es el a veces.

Con respecto a conocer sobre los sujetos actores de la prevención de la violencia, los tres actores educativos consideran que se deben inmiscuir en este propósito maestros, padres de familia y los mismos estudiantes, esta percepción es compartida por $100 \%$ de los maestros y padres de familia; mientras que los estudiantes consideran que la responsabilidad debería de ser de los maestros y estudiantes en un $100 \%$, solo un $67 \%$ de estudiantes considera necesario la participación de los padres de familia, en este mismo ámbito consideran importante la participación y apoyo de otros profesionales para trabajar la prevención de la violencia en la escuela.

Según percepción del $100 \%$ de los directores y el 73\% de los maestros resaltan que no existe una preparación para prevenir y enfrentar la violencia en la unidad educativa a manera de prevención, por esto se realizan intervenciones cuando se presenta alguna situación de violencia o la intervención del hecho fáctico; en contraposición un $27 \%$ de maestros considera haber tenido alguna capacitación en eventos externos a la unidad educativa.

En lo que se refiere a los padres y madres de familia un $97 \%$ afirman no haber tenido oportunidad de capacitarse y los estudiantes en un 91\%, solo un $9 \%$ de los estudiantes resalta el trabajo realizado por los maestros del área de Religión quien generalmente les recomienda para que se respeten. Pero todos coinciden que si existe un cuaderno de registros donde se escribe los actos de violencia, el mismo que sirve para ver la recurrencia y mostrar así a los padres sobre la conducta de los estudiantes (Ver tabla 3 y gráfico 3 ). 
Tabla 3. Competencias en estrategias para abordar la violencia escolar

$\%$ Competencias en estrategias para abordar la violencia escolar

\begin{tabular}{lcccc}
\hline & Directivos & Docentes & Estudiantes & Padres \\
Si tiene & & 27 & 9 & 3 \\
\hline No tiene & 100 & 73 & 91 & 97 \\
\hline
\end{tabular}

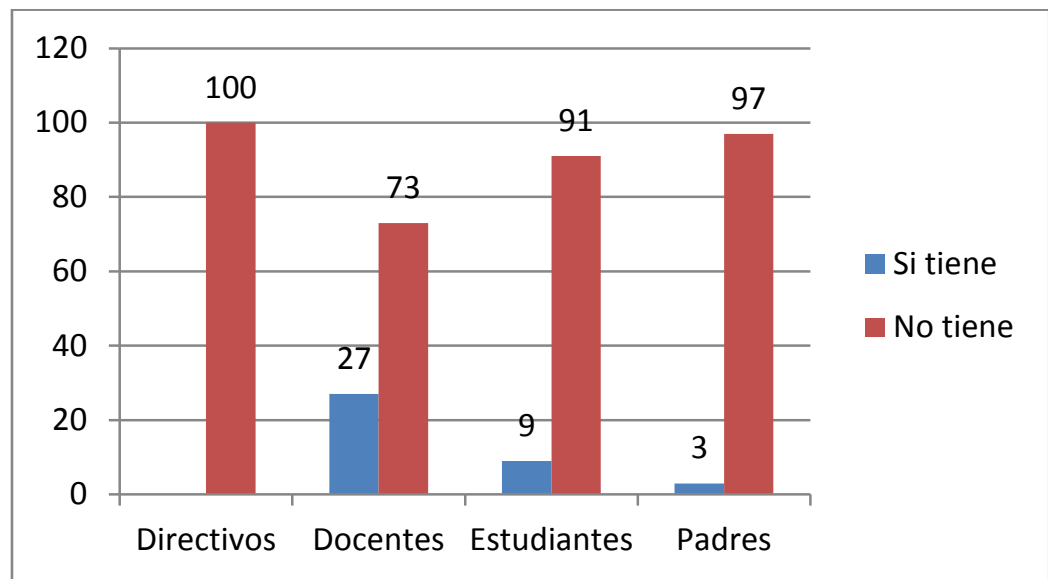

Gráfico 3. \% de competencias para abordar la violencia escolar

Los resultados muestran que maestros y estudiantes y padres de familia están en la disposición de participar en espacios de formación en prevención de la violencia en el ámbito escolar considerando que es una necesidad desde todos los grupos.

Con base a los resultados del diagnóstico se diseña la estrategia pedagógica para la prevención de la violencia escolar desde la educación para la paz en estudiantes del tercero de Educación Secundaria Comunitaria Productiva.
PROPUESTA

La propuesta quedó estructurada en las siguientes partes: (a) Fundamentos sociológicos, psicológicos, pedagógicos $\mathrm{y}$ jurídicos; (b) Objetivos; (c) Metodología fases de diagnóstico, organización, planificación, ejecución y evaluación.

Como puede observarse en el siguiente esquema. 


\section{DIAGRAMA DE LA PROPUESTA DE ESTRATEGIA PEDAGOGICA PARA LA PREVENCION}

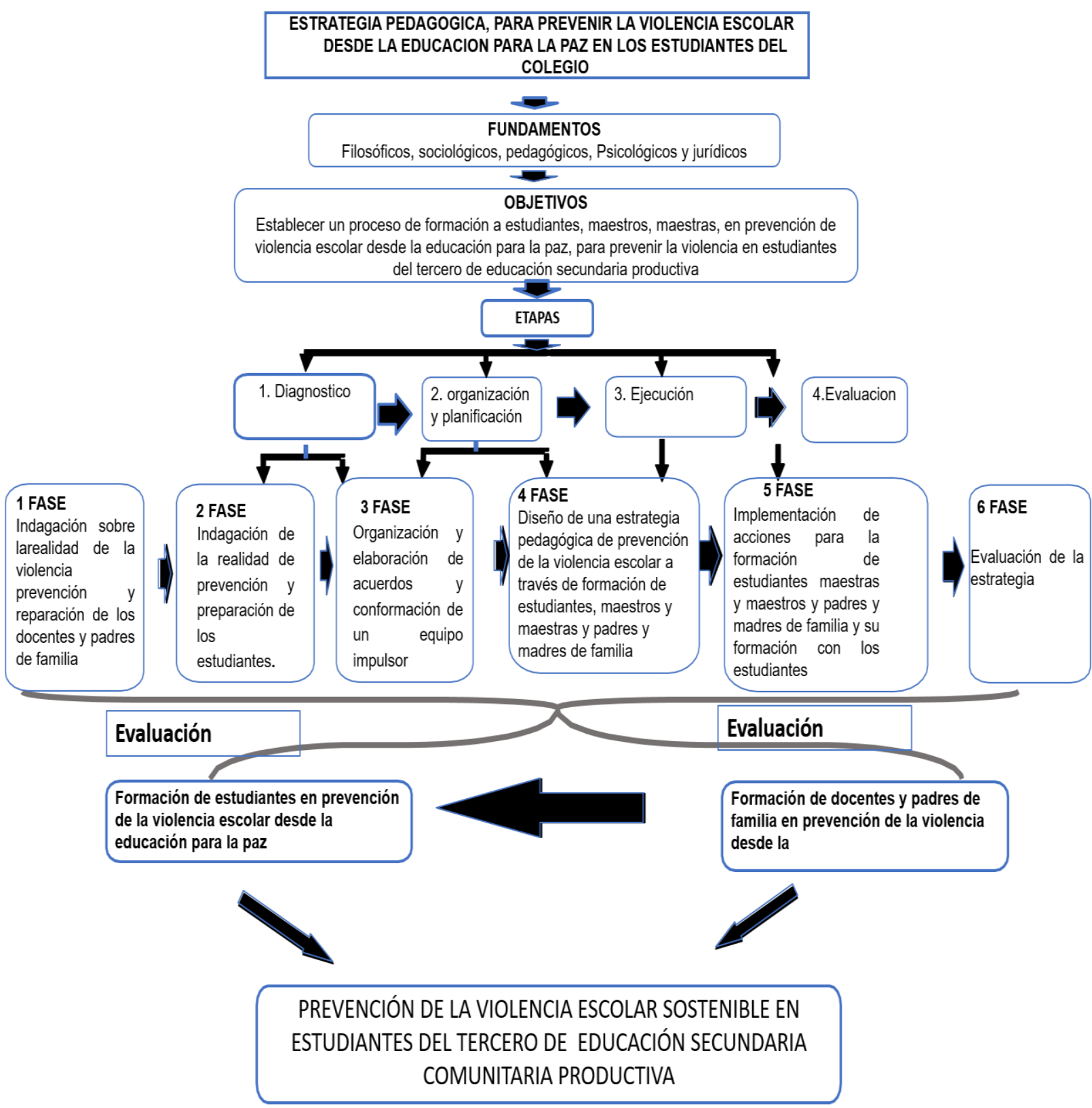


La estrategia pedagógica basada en la educación para la paz prevé actividades de acción abierta cuya estructuración pretende redefinir las relaciones que se establecen en las interacciones personales, así como entre estudiantes- docentes y padres de familia tanto dentro del aula como fuera de ella constituyendo grupos heterogéneos y de cooperación. Las actividades que se sugieren son:

- Exposición de fotografías problematizadas sobre la violencia y sus consecuencias así como las formas de prevención, las mismas que inciten comentario y reflexión, en todos los actores y que las mismas serán analizadas entre los estudiantes en el aula bajo la guía del docente y en el ambiente familia bajo la guía de los padres de familia, esta actividad será realizada una vez por semana.

- Implementación de lecturas sobre valores orientados a la convivencia e interacción pacifica

- Video debate el mismo que estará organizado por los maestros con la participación de los estudiantes y padres de familia.
- Feria educativa "Aprendamos a vivir en paz para Vivir bien y en una cultura de paz"

- Implementación de acciones institucionales concretas para mejorar el clima institucional y organizacional de tal manera se genere mayores espacios de relación, motivación y reconocimiento individual y comunitario, así como de significación de límites por las autoridades y maestros.

Estas actividades estarán orientadas a la formación teórica - práctica que permitirá la interacción entre maestros, estudiantes, directivos y padres de familia.

Por otro lado se implementará un sistema de talleres como una alternativa efectiva para la preparación de los actores educativos. Los talleres como estrategia pedagógica permiten la reflexión individual y colectiva sobre las consecuencias de la violencia y posibilitan la búsqueda de soluciones en correspondencia a las necesidades de los participantes.

Los talleres tendrán una duración de dos horas dos veces al mes en el siguiente cuadro se resume la panificación de las temáticas para cada grupo participante.

Cuadro 1. Cronograma de aplicación de taller 


\begin{tabular}{|c|c|c|c|c|}
\hline $\mathbf{N}^{\circ}$ & Temáticas & $\begin{array}{l}\text { Sesión con los } \\
\text { estudiantes }\end{array}$ & $\begin{array}{l}\text { Sesión con los } \\
\text { docentes }\end{array}$ & $\begin{array}{l}\text { Sesión con } \\
\text { los padres } \\
\text { de familia }\end{array}$ \\
\hline 1 & $\begin{array}{l}\text { De } \\
\text { sensibilización } \\
\text { de la violencia }\end{array}$ & 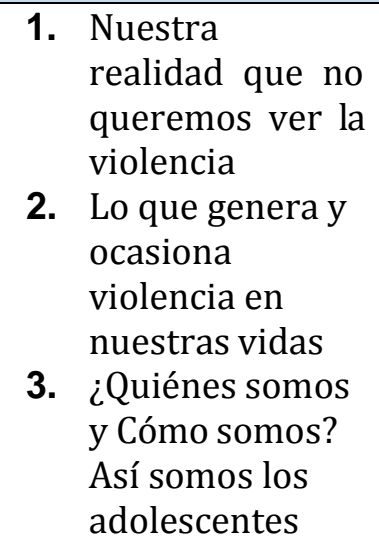 & $\begin{array}{l}\text { 1. La violencia un } \\
\text { problema que nos } \\
\text { afecta } \\
\text { 2. Políticas educativas } \\
\text { que contribuyen a } \\
\text { la prevención de la } \\
\text { violencia escolar y } \\
\text { la educación para la } \\
\text { paz y los derechos } \\
\text { humanos } \\
\text { 3. Consecuencias y } \\
\text { límites }\end{array}$ & $\begin{array}{l}\text { 1. La violencia y } \\
\text { tipos de } \\
\text { violencia } \\
\text { 2. Conociendo a } \\
\text { nuestros hijos } \\
\text { los } \\
\text { adolescentes }\end{array}$ \\
\hline 2 & $\begin{array}{l}\text { Prevención de } \\
\text { la violencia }\end{array}$ & $\begin{array}{l}\text { 4. Comunicación } \\
\text { asertiva. } \\
\text { 5. Aprendiendo a } \\
\text { expresar lo que } \\
\text { pensamos } \\
\text { 6. Mi libertad } \\
\text { acaba donde } \\
\text { empieza la } \\
\text { tuya } \\
\text { 7. Me respeto te } \\
\text { respeto, nos } \\
\text { respetamos }\end{array}$ & $\begin{array}{l}\text { 4. Comunicación } \\
\text { asertiva } \\
\text { 5. Uso adecuado de } \\
\text { poderes del } \\
\text { profesor } \\
\text { 6. Estrategias de } \\
\text { disciplina positiva }\end{array}$ & $\begin{array}{l}\text { 3. Prácticas de } \\
\text { crianza. } \\
\text { 4. Estrategias de } \\
\text { disciplina } \\
\text { positiva }\end{array}$ \\
\hline 3 & $\begin{array}{l}\text { Educación } \\
\text { para la paz, la } \\
\text { convivencia y } \\
\text { la armonía } \\
\text { escolar }\end{array}$ & $\begin{array}{l}\text { 8. } \begin{array}{l}\text { resolvemos } \\
\text { pacíficamente } \\
\text { nuestro } \\
\text { problemas }\end{array} \\
\text { 9. Aprendiendo } \\
\text { a convivir en } \\
\text { paz desde los } \\
\text { valores socio } \\
\text { comunitarios } \\
\text { 10. Aprendiendo a } \\
\text { crecer en } \\
\text { habilidades } \\
\text { sociales }\end{array}$ & $\begin{array}{l}\text { 7. Educación en } \\
\text { valores } \\
\text { 8. Habilidades } \\
\text { sociales } \\
\text { 9. Educación en } \\
\text { democracia y } \\
\text { derechos } \\
\text { humanos }\end{array}$ & $\begin{array}{l}\text { 5. Aprendiendo a } \\
\text { dialogar en paz } \\
\text { 6. Educación en } \\
\text { valores } \\
\text { 7. Habilidades } \\
\text { sociales, para } \\
\text { convivir en } \\
\text { familia y } \\
\text { comunidad } \\
\text { 8. Educación en } \\
\text { democracia y } \\
\text { derechos }\end{array}$ \\
\hline
\end{tabular}

Se planifican 10 sesiones para los estudiantes 9 para los educadores y 8 para los 
padres de familia.

La propuesta tiene instrumentos para evaluar cada una de las actividades al finalizar cada sesión, lo que permitirá el reajuste de alguna actividad que no cumpla con el objetivo establecido.

En la evaluación se debe tomar en cuenta la experiencia de los participantes, resaltar logros, reflexionar sobre los errores y aclarar las dudas. Como criterios de evaluación se debe asumir la participación, responsabilidad, cooperación, trabajo en equipo, posición crítica y reflexiva sobre la implementación de las acciones y las temáticas de los talleres, así como el efecto de transformación que generó la actividad a nivel cognitivo, afectivo, actitudinal y conductual, expresado en la forma de ser y actuar del participante.

\section{CONCLUSIONES}

El diagnóstico realizado a través de la implementación de métodos empíricos e instrumentos permitió evaluar el fenómeno de la violencia en la escuela constándose la presencia de la violencia en sus distintas formas manifestaciones y causas, en ella prevalece la existencia de violencia psicológica, seguida de la violencia física.

También permitió develar que no existen prácticas de prevención de la violencia desde la educación para la paz, sino simples intervenciones sobre el hecho fáctico, A esto se suma la escasa preparación para este propósito tanto de maestros padres de familia y estudiantes lo que evidencia el limitado desarrollo de las habilidades para establecer relaciones sociales y una convivencia armónica entre los miembros de la comunidad.

La estrategia pedagógica para la prevención de la violencia escolar desde la educación para la paz, sustentada en una concepción ecológica comunitaria y da cuenta que es una buena alternativa en la preparación de los maestros y padres de familia quienes se constituyen en los mediadores significativos que pueden incidir de manera comunitaria en la prevención de la violencia desde la educación para la paz en los estudiantes del tercero de educación secundaria

La implementación de manera parcial de la estrategia pedagógica, permite determinar que la propuesta es factible de aplicar y efectiva para atender la prevención de la violencia escolar desde la educación para la paz.

\section{Recomendaciones}

La Universidad y las Unidades Educativas deben seguir promoviendo investigaciones sobre la prevención de la violencia escolar, con el propósito de establecer una convivencia armoniosa, esto les permitiría cumplir el mandato constitucional, de implementar un plan de acción para prevenir y disminuir la violencia escolar y promover una educación basada en una cultura de paz.

Las Unidades Educativas deben atender incluir la formación de todos los actores educativos de manera continua para hacer sostenible la prevención y así desarrollar una cultura de paz.

Esta propuesta puede ser socializada en otras Unidades Educativas del contexto Potosino para generar una red que le de atención a la prevención de la violencia en el contexto escolar.

Seguir con futuras investigaciones para mejorar o perfecciona la presente estrategia pedagógica

Por último se hace necesario implementar de procesos educativos adecuados que posibiliten la prevención de la violencia escolar, es decir a partir de la 
implementación de la educación para la paz que permita difundir una cultura de paz, reconocer la diversidad, promover la tolerancia, la negociación, la concertación y el diálogo a partir del respeto a los derechos de las personas en la escuela y con incidencia en toda la comunidad.

\section{REFERENCIAS}

Bronfenbrenner, U. (1987). La ecología del desarrollo humano. Argentina: Paidós.

Díaz Aguado (2002) Por una cultura de la convivencia democrática. Revista Interuniversitaria de Formación del Profesorado, (44), 55-78

Díaz Aguado, M. (2005) Por qué se produce la violencia escolar y cómo prevenirla. Revista Iberoamericana de Educación, (37), 17-47

Díaz-Barriga, F. y Hernández, G. (2002). Estrategias docentes para un aprendizaje significativo. Una interpretación constructivista. (2a. ed.) México: McGrawHill Interamericana

Estado Plurinacional de Bolivia (2009) Constitución Política del Estado. Bolivia: Autor

Estado Plurinacional de Bolivia (2012). Decreto Supremo 1302. Bolivia: Autor.

García, M., y Madriaza, P. (2005). Lineamientos para la intervención en violencia escolar en Chile. Revista Persona y Sociedad, 19(1), 135-154

Huanacuni, F. (2010). Buen vivir/ vivir bien. Filosofía, políticas, estrategias $\mathrm{y}$ experiencias regionales andinas. La Paz Bolivia: Coordinadora Andina de Organizaciones Indígenas

Ministerio de Educación (2015) Unidad de formación $N^{\circ} 1,2,3,4,5,6,7,8$,9. Modelo Educativo Socio comunitario Productivo.
Cuaderno de Formación Continua. Equipo PROFOCOM. La Paz, Bolivia: Autor.

Ministerio de Educación y Ciencia de España (1992): Educación para la Paz. Temas Transversales. Madrid: Autor.

Mora-Merchán, J. A. (2001). El fenómeno Bull ying en las escuelas de Sevilla. Tesis doctoral. ficha-resumen. Revista Interuniversitaria de Formación del Profesor. (41)

Niño Rojas, V. (2011). Metodología de la investigación. Diseño y ejecución. Colombia: Ediciones de la U.

Ortega, R. (2001). Violencia interpersonal en los centros educativos de enseñanza secundaria. Un estudio sobre malos tratos e intimidación entre compañeros. Escuela como centro de cambio. Revista de Educación, 304. Centro de Publicaciones de MEC.

Oteros, A. (2006). La agresividad como conducta perturbadora en el aula. Revista Digital Investigación y Educación [Revista en línea] 26(3), Disponible: http://www.csicsif.es/andalucia/module s/mod_sevilla/archivos/revistaense/n26 /26080151.pdf [Consulta: 2014, Noviembre 14]

Rodney, Y. (2010). Estrategia Pedagógica dirigida a la preparación del profesorado para la prevención de la violencia escolar. Tesis doctoral, no publicada, Universidad José Enrique Varona, La Habana.

Torrego, J. y Negro, A. (2012). Aprendizaje cooperativo en las aulas. Fundamentos y recursos para su implantación. España: Alianza

UNESCO (2016). Documento del programa para una cultura de paz. Paris, Francia: Autor 